\title{
Pulsar rotation measures and the large-scale magnetic structure of the Milky Way
}

\author{
Charlotte Sobey, Aristeidis Noutsos \& Michael Kramer \\ Max-Planck-Institut für Radioastronomie, Auf dem Hügel 69, 53121 Bonn, Germany \\ E-mail: csobey@mpifr-bonn.mpg.de, anoutsos@mpifr-bonn.mpg.de, \\ mkramer@mpifr-bonn.mpg.de
}

\begin{abstract}
Faraday rotation measures (RMs) of polarised pulsar emission and extragalactic sources present the most efficient observable for determining the configuration of the magnetic field of the Galaxy, both in its plane and at high latitudes. Reconstructions of the Galactic magnetic field (GMF) allow a deeper understanding of numerous astrophysical processes in the interstellar medium and even of the cosmological microwave background. LOFAR (the LOw Frequency ARray) will soon embark on an all-sky pulsar survey, which will return hundreds of new pulsar discoveries. In addition, the High Time Resolution Universe survey is also currently searching for pulsars, at higher frequencies. The subsequent RM data from any pulsars discovered in these surveys, together with the current sample, will provide unprecedented coverage of the Galactic plane and halo. We present our plans to select an appropriate sample of pulsar RMs for use with the powerful method of wavelet tomography. In addition, we show first simulations of how additional RM values improve the wavelet-based reconstruction of the kpc-scale GMF.
\end{abstract}

25th Texas Symposium on Relativistic Astrophysics

December 6-10, 2010

Heidelberg, Germany 


\section{The Galactic Magnetic Field}

Magnetic fields play a crucial role in many astrophysical processes within galaxies. These include, but are not limited to, deflection of ultrahigh energy cosmic rays (UHECRs), evolution of molecular clouds, star formation and hydrostatic balance in the interstellar medium (ISM).

Although our Galaxy provides a uniquely detailed case for studies of magnetic fields [1], the structure of the Galactic magnetic field (GMF) remains largely unexplored. The GMF structure is often modelled as the sum of a regular, large-scale component, coherent over kpc scales and small-scale turbulent fields with characteristic scales of 10-100 pc. The turbulent components can be attributed to fields of localised structures including H II regions and supernova remnants (SNRs), i.e. the Gum Nebula and North Polar Spur. Figure 1 depicts three, of many, speculative theoretical models of the large-scale GMF, which are often used for comparison when analysing observational data.

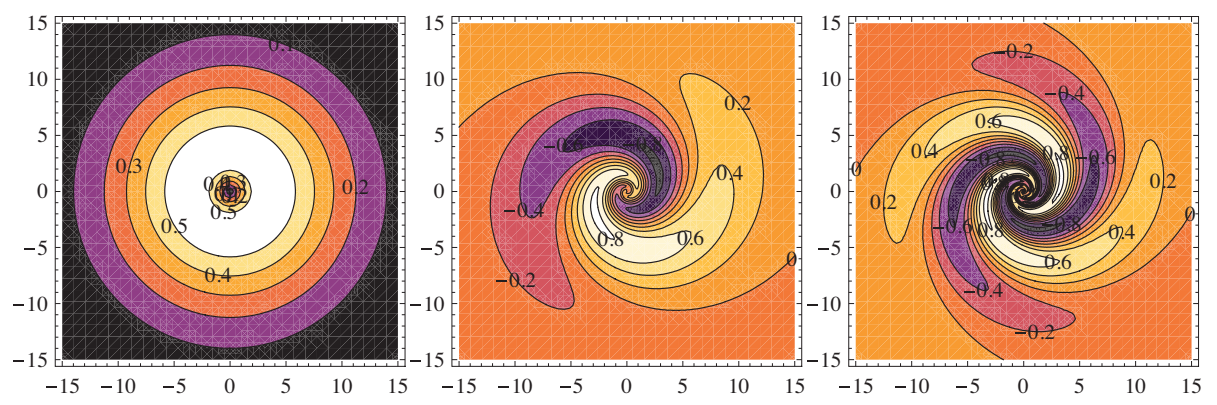

Figure 1: Examples of the product of modelled regular magnetic field strength and thermal electron density in the Galactic mid-plane. Azimuthal field modes $m=0,1,2$ (axisymmetric, bisymmetric and quadrisymmetric spiral fields respectively) are shown left to right. From [2].

\section{Pulsar Rotation Measures}

Many observational tracers of the ISM have been used in the attempt to determine the strength and direction of the GMF. Examples of such methods are described below:

- Polarisation of optical starlight due to scattering by dust grains preferentially aligned along local magnetic field lines. This provides the sky-projected magnetic field component averaged over the integrated size of the scattering screen between the star and observer. Magnetic fields are weighted by the intervening dust, which is usually unknown, and measurements are only currently possible out to $2-3 \mathrm{kpc}$ from the Sun. Despite this, maps of the GMF have been produced from polarisation observations of several thousands of stars, i.e. [3].

- Linearly polarised thermal emission from dust in $\mathrm{mm}$, sub-mm and IR wavelengths preferentially aligned along local magnetic field lines. This also produces the sky-projected component of the magnetic field, each wavelength band probing the distribution in grain size. Magnetic fields coherent over $\sim 1-10 \mathrm{pc}$ can be detected, allowing observations of the 
large-scale GMF. Although in the future this method may become more sensitive, perhaps even to the magnetic fields of nearby galaxies, this is only currently possible for dense, bright regions in the Galaxy, i.e. the central molecular cloud ring [4].

- Zeeman splitting of spectral lines produced in emission or absorption regions. This method can measure the parallel component of the magnetic field. Amplitudes on the order of $m \mathrm{G}$ are generally required, in comparison to the significantly weaker $\mu \mathrm{G}$ field of the large-scale GMF. Observations are hence currently confined to masers and molecular clouds. However, where measurements are spread across the Galactic plane, trends in the overall direction of the detected fields resemble the large-scale GMF deduced via alternative methods i.e. [5].

- Polarisation observations of diffuse radio synchrotron emission produced by the Galactic population of cosmic-ray (relativistic) electrons. This method provides the sky-projected, transverse magnetic field. The strength of the ordered GMF can also be estimated, assuming energy equipartition between cosmic rays and the total magnetic field [6]. Typical large-scale GMF values deduced are likely to be higher than from other methods due to the contribution of the small-scale, turbulent component to the measured field.

An alternative method to those presented above uses the Faraday rotation of polarised pulsars and extragalactic sources. This has also been successfully used to derive the magnitude and direction of the GMF (i.e. [1], [7], [8]).

Electromagnetic waves propagating through a magnetised medium are subject to Faraday rotation, where the plane of linear polarisation rotates between the points of emission and observation. The effect is frequency dependant, causing progressively longer wavelengths $(\lambda)$ to experience increasing rotation of the polarisation angle $(P A)$ :

$$
\Delta P A=R M \lambda^{2} .
$$

The rotation measure $(\mathrm{RM})$ is directly related to the number density of plasma electrons, $n_{\mathrm{e}}$, and the magnetic field of the plasma, $\mathbf{B}$, integrated along a unit column of length equal to the distance to the pulsar, $\mathrm{d}$ :

$$
R M=\kappa \int_{0}^{\mathrm{d}} n_{e}(s) \mathbf{B}(s) \cdot d \mathbf{s}
$$

where $d \mathbf{s}$ is the path vector element in the direction of wave propagation. Expressing $n_{e}$ in $\mathrm{cm}^{-3}$, B in $\mu \mathrm{G}$, $\mathrm{d}$ in pc and the constant $\kappa$ equal to $0.812 \mathrm{rad} \mathrm{m}^{-2} \mathrm{~cm}^{3} \mu \mathrm{G}^{-1} \mathrm{pc}^{-1}$ to three significant figures, the RM is measured in the usual units of $\mathrm{rad} \mathrm{m}^{-2}$. The magnetic field parallel to the LOS from the pulsar toward the observer, $\left\langle B_{\|}\right\rangle$, can be determined when combined with the Dispersion Measure (DM),

$$
\left\langle B_{\|}\right\rangle=\frac{\int_{0}^{\mathrm{d}} n_{e}(s) \mathbf{B} \cdot d \mathbf{s}}{\int_{0}^{\mathrm{d}} n_{e}(s) d s}=1.232 \frac{R M}{D M}
$$

where the units of DM are given in $\mathrm{pc} \mathrm{cm}^{-3}$ and $\left\langle B_{\|}\right\rangle$in $\mu \mathrm{G}$.

The advantages of using polarised pulsar emission to measure both the Galactic planar and halo fields include:

- Pulsed pulsar emission typically carries a high degree of linear polarisation, for example see Figure 2, allowing RM measurements to be easily obtained. 
- Observed RM values are a direct consequence of the ISM. Pulsar magnetospheres are populated by electron-positron pairs, resulting in zero net Faraday rotation.

- The distribution of pulsars throughout the Galactic volume allows three-dimensional sampling over many LOS and directions. Dense concentrations in the galactic plane, especially along spiral arms, provide a good sample of the planar GMF. Pulsars at higher latitudes can also be combined with extragalactic RM values to sample the GMF in the halo.

- Combining the pulsar DM with a model of the Galactic free-electron density provides a direct estimate of the pulsar distance, useful for producing GMF image maps, i.e. see Figure 3.

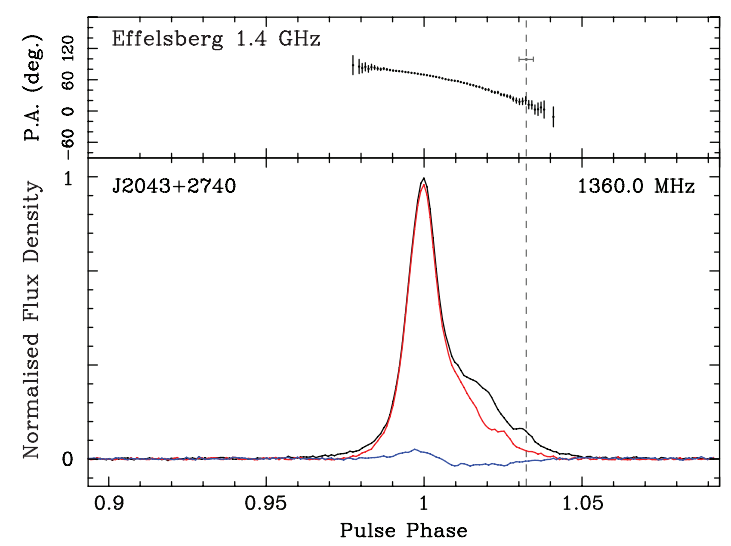

Figure 2: Polarisation profile of PSR J2043+2740 obtained with the 7-beam, 20-cm receiver and pulsar digital filterbank at the Effelsberg Radio Telescope. Black, red and blue components show total, linear polarisation and circular polarisation intensity respectively. From [9].

An example image map depicting the projected value of the parallel component of the GMF in the Galactic disk, obtained using archival pulsar RM data, is shown in Figure 3.

One of the latest analyses of RM estimates for pulsars and extragalactic sources in the fourth Galactic quadrant led to a picture in which the magnetic field of the Galaxy has a clear large-scale structure of $\sim$ a few $\mu \mathrm{G}$, organised along logarithmic spiral arms [10]. There appears to be clear field reversals from the Norma arm to the Norma-Crux interarm region and from the Norma-Crux interarm region to the Crux arm. The best fit model of the large-scale field most closely resembles the quadrisymmetric spiral (QSS) of [2], see Figure 1. The small-scale field superimposed on this large-scale field is estimated to be at least as strong on scales $\lesssim 1 \mathrm{kpc}$. The process which forms a GMF with such configuration, i.e. many reversals where large and small scales coexist, remains a largely unanswered question.

Limitations of using pulsars to measure the Galactic and halo fields include:

- The simple approach of dividing pulsar RM by DM implicitly assumes constant, average values of $n_{e}$ and $\left\langle B_{\|}\right\rangle$. This is known to be unrealistic especially following evidence of field reversals between arm and inter-arm regions. 


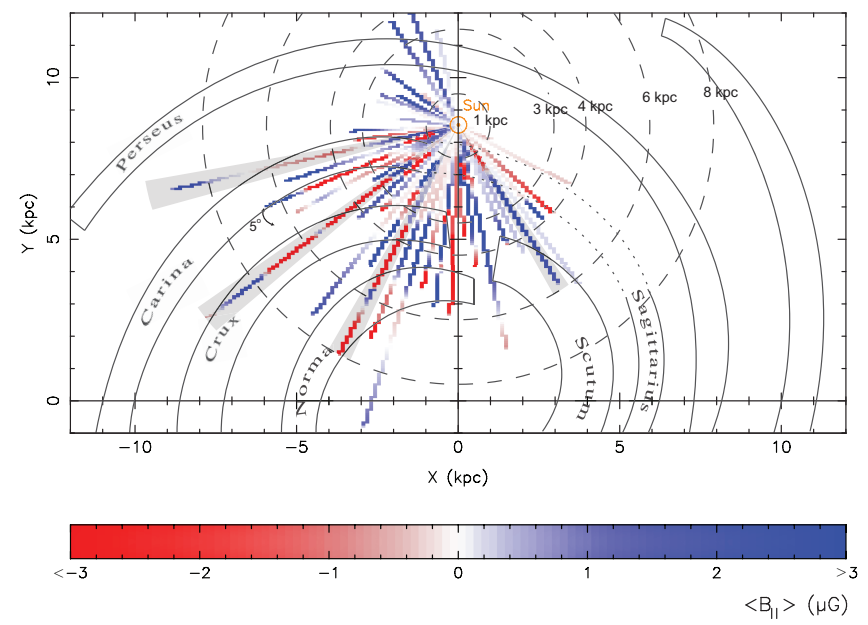

Figure 3: A map of the projected $\left\langle B_{\|}\right\rangle$values using RM and DM data. Each pixel does not correspond to an individual pulsar, but to a linear interpolation over the data, binned in 72 five-degree longitude sectors and 1000 ten-pc distance bins. Field values are represented between +3 and $-3 \mu \mathrm{G}$ in hues of blue and red where directed towards and away from the Sun respectively. The four sectors shaded grey indicate the directions of spiral arms and hence possible GMF reversals in the plane. From [8].

- Pulsar distances, estimated using DM values and a model of $n_{\mathrm{e}}$ [11], are typically subject to uncertainties of $\sim 20 \%$. Hence, it is important to use independently determined distances where possible. For example, annual parallax, H I absorption, association with globular clusters or pulsar timing, i.e. [12].

- It is desirable to eliminate RM values where the LOS coincides with localised, small scale structures which introduce noise, i.e. towards H II regions and SNRs [10]. Nevertheless, this noise can be filtered out by introducing a wavelet-based description of the RM sample, as explained in the following.

\section{Wavelet Analysis}

Pulsar RM and DM values may be analysed using the method of wavelet tomography in order to reconstruct the large-scale GMF [13]. Specialised wavelets can be devised to:

- Filter out the noise in RM data caused by small-scale fluctuations of the ISM and unequal spacing of data points.

- Minimise noise amplification in the analysis of RMs and DMs, represented by integrations along the LOS, given the range in distances at which the localised GMF is sampled.

Figure 4 displays an example reconstruction of a GMF mid-plane model using wavelet tomography and the positions of known pulsars with RM data. Neither ad-hoc assumptions about the GMF structure nor model-fitting are included. This method has been shown to work well with regularly distributed or randomly scattered data where gaps do not exceed one half the scale of the 

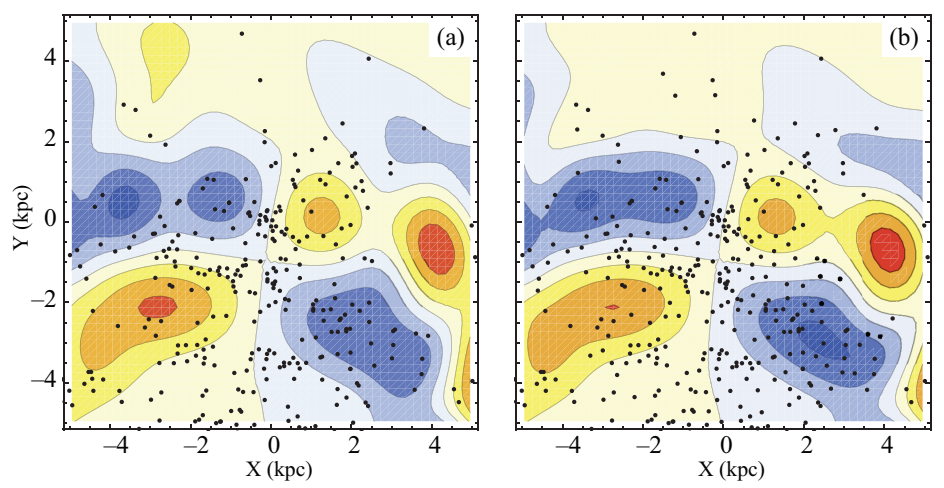

Figure 4: Simulated reconstruction of a model large-scale magnetic field using; (a) the sample of existing pulsar RM values and (b) those in (a) plus 50 additional RM values in sparsely sampled locations. Black dots represent pulsar positions, the Sun at the origin. Hues of blue and red represent where the field is directed towards and away from the observer respectively. From R. Stepanov (private communication).

wavelet. For scales of $1.5 \mathrm{kpc}$ however, the RM data currently catalogued for pulsars becomes too sparse beyond $3 \mathrm{kpc}$ of the Sun. The improvement in reconstructing the model GMF, through including a small number of additional pulsar RM values located in poorly sampled areas, is illustrated in Figure 4. Enhanced contrast and greater elongation of structures are clearly visible.

The fraction of pulsars with measured RMs currently stands at a little over $40 \%$. Increasing this statistic is therefore highly desirable for the purpose of wavelet analysis of the GMF. Such polarisation observations are also supplementary for other studies into, for example, pulsar emission geometry and orientation.

\section{Future Prospects}

Polarisation observations of known pulsars without RM data and also of pulsars discovered in future surveys will inevitably improve the RM sampling of the GMF.

Observations of catalogued pulsars located in poorly sampled regions in quadrants I and II of the Galaxy are currently planned with the Effelsberg Radio Telescope.

Several searches for undiscovered pulsars are ongoing or are due to commence in the near future. The High Time Resolution Universe (HTRU) survey currently under way, using both Effelsberg and Parkes Radio Telescopes, promises to deliver several new discoveries in the Galactic disk, i.e. [14] and [15].

The new LOFAR radio telescope is also currently being commissioned. This presents an exciting prospect for discovering many new pulsars at low frequencies, mainly due to the large collecting area and wide field of view. A Galactic survey over a 60-day period at $140 \mathrm{MHz}$ has the potential to return over a thousand new pulsars [16], see Figure 5.

With new discoveries from increasingly sensitive telescopes planned for the future (i.e. FAST, SKA) the number of pulsars with RM data is destined to improve, paving the way for much exciting science and specifically, greater accuracy in the reconstruction of the GMF. 

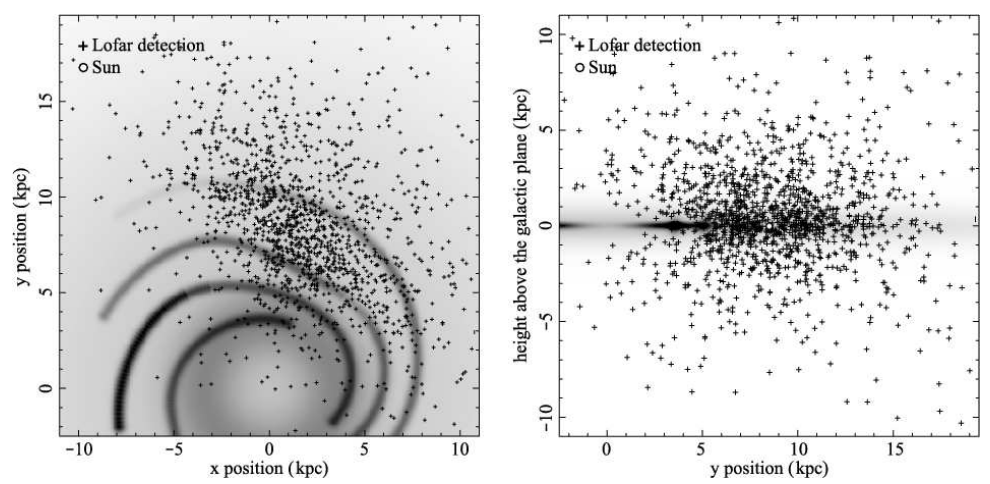

Figure 5: Discoveries of $>1000$ pulsars predicted from simulations of a 60-day LOFAR all-sky survey, projected on the Galactic plane (left) and through the Galactic disk (right). From [16].

\section{References}

[1] J. L. Han, in The Magnetized Interstellar Medium, eds. B. Uyaniker, W. Reich and R. Wielebinski, Copernicus GmbH, Katlenburg-Lindau, 2004, p., [astro-ph / $0402170 v 1$ ]

[2] R. Stepanov, T. G. Arshakian, R. Beck, P. Frick and M. Krause, A\&A 480, 45 (2008) [astro-ph/0711.1267v3].

[3] P. Fosalba, A. Lazarin, S. Prunet and J. A. Tauber, ApJ 564, 762 (2002) [a st ro-ph/ $0105023 v 2]$.

[4] D. T. Chuss, J. A. Davidson, J. L. Dotson, C. D. Dowell, R. H. Hildebrand, G. Novak and J. E. Vaillancourt, ApJ 599, 1116 (2003).

[5] J. L. Han and J. S. Zhang, A\&A 464, 609 (2007) [astro-ph/ $0611213 v 3$ ].

[6] X. H. Sun, J. L. Han, W. Reich, P. Reich, W. B. Shi, R. Wielebinski and E. Fürst, A\&A 463, 993 (2007) [astro-ph/0611622v1].

[7] J. C. Brown, M. Haverkorn, B. M. Gaensler, A. R. Taylor, N. S. Bizunok, N. M. McClure-Griffiths, J. M. Dickey and A. J. Green, ApJ 663, 258 (2007) [astro-ph/ 0704 . 04 58v1].

[8] A. Noutsos, S. Johnston, M. Kramer and A. Karastergiou, MNRAS 386, 1881-1896 (2008) [astro-ph/0803.0677v1].

[9] A. Noutsos, et al., ApJ 728, id.77 (2011) [astro-ph/1012 . 4658v1].

[10] T. Nota and P. Katgert, $A \& A$ 513, id.A65 (2010).

[11] J. M. Cordes and T. J. W. Lazio, (2002) astro-ph/0207156.

[12] J. P. W.Verbiest, D. R. Lorimer and M. A. McLaughlin, MNRAS 405, 575-580 (2010) [astro-ph/1002.1213v2].

[13] R. Stepanov, P. Frick, A. Shukarov and D. Sokoloff, A\&A 391, 361 (2002) [ast ro-ph/ 0112507 ].

[14] M. J. Keith, A. Jameson, W. van Straten, M. Bailes, S. Johnston, M. Kramer, A. Possenti, S. D. Bates, N. D. R. Bhat, M. Burgay, S. Burke-Spolaor, N. D’Amico, L. Levin, P. L. McMahon, S. Milia and B. W. Stappers, MNRAS 409, 619 (2010) [astro-ph/1006.5744v2].

[15] C. Ng, E. Barr, et al., in proceedings of Texas 2010, PoS ( Texas 2010) 066.

[16] J. van Leeuwen and B. W. Stappers, $A \& A$ 509, id.A7 (2010) [astro-ph/0910 . 5118v1]. 